\title{
COMPACTIFICATION AND COMPLETION AS ABSOLUTE CLOSURE
}

\author{
ANTHONY W. HAGER
}

\begin{abstract}
It is well known that a Tychonoff (respectively, Hausdorff uniform) space is compact (resp., complete) iff it is absolutely closed, i.e., dense in no other such space; we shall sketch a proof of this to our purpose, which is: Given $X$, we apply Zorn's Lemma to obtain a space maximal with respect to the property of containing $X$ densely, thus compact or complete. For uniform spaces, the "maximal extension" is automatically the completion; for Tychonoff spaces, we must, and do, explicitly arrange things so that the "maximal extension" has the universal mapping property describing the Stone-Cech compactification. A variant of the construction yields the Hewitt realcompactification. A crucial point in the proofs is (of course) the exhibition of an upper bound for a chain; this is, in essence, a direct limit construction.
\end{abstract}

All topological spaces will be Tychonoff (i.e., completely regular Hausdorff), and all uniform spaces Hausdorff. Statements about topological spaces, will be said to be "in Tych", and about uniform spaces, "in Unif". $X \in$ Tych (resp., Unif) is called absolutely closed in Tych (resp., Unif) if any dense embedding $X \stackrel{e}{\rightarrow} Y, Y \in$ Tych (resp., Unif) is onto. For $X \in \mathrm{Tych}, C^{*}(X)$ is the set of bounded continuous real-valued functions on $X$, and $X$ is $C^{*}$-embedded in $Y$ means there is an embedding $X \stackrel{e}{\rightarrow} Y$ with each $f \circ e^{-1}\left(f \in C^{*}(X)\right)$ extending continuously over $Y$ (see [4]). We shall use the covering description of uniform spaces (per [7]).

Only (b) of the following is used in the Theorem.

Lemma. (a) A complete (resp., compact) space is absolutely closed.

(b) If $X$ is not complete (resp., compact), then $X$ is densely and properly embedded (resp., $C^{*}$-embedded) in another space.

Proof. (a) If $X$ is not closed in $Y$, and $p \in \bar{X}-X$, then the filter of intersections with $X$ of $Y$-neighborhoods of $p$ does not cluster in $X$. Thus: in Tych $X$ is not compact; in Unif, the filter is Cauchy and $X$ is not complete.

Received by the editors June 22, 1972.

AMS (MOS) subject classifications (1970). Primary 54D35, 54E15.

Key words and phrases. Compactification, completion, absolutely closed.

(c) American Mathematical Society 1973 
(b) In Unif: Suppose $X$ has the nonconvergent Cauchy filter $\mathscr{F}$. Let $Y=X \cup\{\mathscr{F}\}$, with uniformity of covers $\overline{\mathscr{U}}$, where $\mathscr{U}$ is $X$-uniform, defined by: $\overline{\mathscr{U}}=\{U: U \in \mathscr{U}$ and $U \notin \mathscr{F}\} \cup\{U \cup\{\mathscr{F}\}: U \in \mathscr{U} \cap \mathscr{F}\}$. One readily checks the axioms for a uniformity, the Hausdorff property, and density of $X$.

In Tych: Given $X$, the family $\mathscr{Z}$ of sets $\{x: f(x)=0\}\left(f \in C^{*}(X)\right)$ is a base for the closed sets. Since "compact relative to a base" implies compact, if $X$ is not compact, there is a filter $\mathscr{F}$ of sets in $\mathscr{Z}$ with $\cap \mathscr{F}=\varnothing$. We take $\mathscr{F}$ to be an ultrafilter. Again, let $Y=X \cup\{\mathscr{F}\}$. For $f \in C^{*}(X)$, we shall define $\bar{f}: Y \rightarrow R$ and then give $Y$ the weak topology generated by $\left\{\bar{f}: f \in C^{*}(X)\right\}$. This forces complete regularity; the Hausdorff property and density of $X$ are easy. So let $f \in C^{*}(X)$; say $f(X) \subset[a, b]$. Given $n$, let $I_{n}$ be a closed subinterval of $[a, b]$, of length $1 / n$, with $f^{-1}\left(I_{n}\right) \in \mathscr{F}$ (exercise). Evidently $\bigcap_{n} I_{n}$ is a point, which we call $\tilde{f}(\mathscr{F})$.

THEOREM. Each uniform (resp., topological) space is densely (resp., densely $C^{*}$ ) embedded in a complete (resp., compact) space.

REMARK. In (b) of the Lemma, what is done is to construct one point, called $\mathscr{F}$, of the completion or Stone-Cech compactification, to be "added" to the given space $X$. For Unif, that $\mathscr{F}$ is a point of the completion follows by application of the universal mapping property of arbitrary extensions of $X$-that is, completion is unique while compactification is not. This focuses on a curious point: The mapping theorem is not used in constructing (below) the complete extension by our method, but the $C^{*}$-embedding in (b) of the Lemma is needed to make the construction work in Tych as is shown by the example from [5] referred to below under "Direct limits".

Proof. In Unif: Suppose $X$ is not complete. Let $\mathscr{P}$ be a set of pairs $(Y, e)$ where $Y$ is a space and $X \stackrel{e}{\rightarrow} Y$ is a dense embedding, with the property that if $X \stackrel{h}{\rightarrow} Z$ is any dense embedding, then there is $(Y, e) \in \mathscr{P}$ and an isomorphism $Z \stackrel{i}{\rightarrow} Y$ with $i \circ h=e$. (If $X \stackrel{h}{\rightarrow} Z$ is a dense embedding, then $|Z| \leqq 2^{2|X|}$. So fix a set $S$ with $|S|=2^{2|X|}$, and let $\mathscr{P}$ be just those $(Y, e)$ 's with $Y$ a subset of $S$.) By the Lemma, $\mathscr{P} \neq \varnothing$. Partially order $\mathscr{P}:(Y, e) \leqq\left(Y^{\prime}, e^{\prime}\right)$ if there is an embedding $Y \stackrel{h}{\rightarrow} Y^{\prime}$ with $h \circ e=e^{\prime}$. One readily sees that such an $h$ is unique. We are to show that chains in $\mathscr{P}$ are upper-bounded. Then Zorn's Lemma will yield a maximal member of $\mathscr{P}$, which is complete, by the Lemma. So let $\mathscr{C}$ be a chain in $\mathscr{P}$. Take the sum $\sum\{Y:(Y, e) \in \mathscr{C}\}$ and factor it by this equivalence relation: if $y_{1} \in Y_{1}$ and $y_{2} \in Y_{2}$, then (say) $\left(Y_{1}, e_{1}\right) \leqq\left(Y_{2}, e_{2}\right)$, so there is unique $Y_{1} \stackrel{h}{\rightarrow} Y_{2}$ with $h \circ e_{1}=e_{2} ; y_{1} \sim y_{2}$ if $h\left(y_{1}\right)=y_{2}$. Call this set $Q$ and let $\sum\{Y:(Y, e) \in \mathscr{C}\} \stackrel{a}{\rightarrow} Q$ be the quotient map. Let $\mu$ be the collection of covers $\mathscr{U}$ of $Q$ such that for each $(Y, e) \in \mathscr{C}, q^{-1}(\mathscr{U} \cap Y$ is uniform in $Y$. 
The axioms for a uniformity are readily checked, with the possible exception of star-refinements: If $\mathscr{U} \in \mu$, define $\mathscr{V} \in \mu$ with $\mathscr{V}^{*}<\mathscr{U}$, as follows. Given $(Y, e) \in \mathscr{C}$, choose $\mathscr{V}(Y, e)$, uniform in $Y$, with $\mathscr{V}(Y, e)^{* *}<q^{-1}(\mathscr{U}) \cap Y$. For $U \in \mathscr{U}$, let

$$
V_{u}(Y, e) \equiv \bigcup\left\{V \in \mathscr{V}(y, e): V^{* *} \subset q^{-1}(U) \cap Y\right\},
$$

$V_{u} \equiv \bigcup\left\{V_{u}(Y, e):(Y, e) \in \mathscr{C}\right\}$, and $\mathscr{V} \equiv\left\{V_{u}: U \in \mathscr{U}\right\}$. So give $Q$ the uniformity $\mu$. If in $Q, p_{1} \neq p_{2}$, then there is $(Y, e) \in \mathscr{C}$ with $q^{-1}\left(p_{1}\right) \cap Y \neq$ $q^{-1}\left(p_{2}\right) \cap Y$; the Hausdorff property follows. Take any $(Y, e) \in \mathscr{C}$; then $h \equiv(q \mid Y) \circ e$ embeds $X$ densely in $Q$ and is independent of $(Y, e)$. Finally, choose $(L, l) \in \mathscr{P}$ for which there is an isomorphism $Q \stackrel{i}{\rightarrow} L$ with $i \circ h=l$; $(L, l)$ is the required upper bound for $\mathscr{C}$.

In Tych: If $X$ is not compact, let $\mathscr{P}$ be a set of pairs $(Y, e)$, where $X \stackrel{e}{\rightarrow} Y$ is a dense $C^{*}$-embedding, such that any dense $C^{*}$-embedding is naturally homeomorphic to a member of $\mathscr{P}$. Take a chain $\mathscr{C}$, and construct $Q$ as above. Without yet topologizing $Q$, construct $X \stackrel{h}{\rightarrow} Q$, and now extend all $f \circ h^{-1}\left(f \in C^{*}(X)\right)$ from the compatible extensions of the $f \circ e^{-1}$ over the $(Y, e) \in \mathscr{C}$. The extensions separate points, so the weak topology on $Q$ is Hausdorff, and of course completely regular. $h$ is an embedding, because by construction it is a $C^{*}$-embedding.

Compactification, realcompactification, and completion. First, the Hewitt realcompactification $v X$ can be constructed in a manner similar to the above construction of the Stone-Cech compactification. Let $C(X)$ be all continuous real-valued functions on $X$, and define $C$-embedding of $X$ in the obvious way. As a definition of realcompact: each ultrafilter $\mathscr{F}$ in $\mathscr{Z}$ (the sets $\{x: f(x)=0\}(f \in C(X))$ with the countable intersection property has $\cap \mathscr{F} \neq \varnothing$. Then the analogue of the Lemma holds, with quite similar proof: $X$ is realcompact iff $X$ is closed whenever $C$-embedded $[4,8 . A]$. (To prove $\Leftarrow$, one uses the Lindelöf property of $R$, as compactness of $[a, b]$ was used previously.) Then, given $X \in$ Tych, $X$ is densely $C$-embedded in a realcompact space, called $v X$, by a Zorn's Lemma argument as above.

Of course, both the compactification and realcompactification theorems can be derived from the completion theorem, as follows. Given $X \in$ Tych, let $\mu$ be the weak uniformity generated by $C^{*}(X)$ (resp., $C(X)$ ), and let $\tilde{X}$ be the completion. Then, (i) $X$ is compact (resp., realcompact) iff $(X, \mu)$ is complete; (ii) all functions in $C^{*}(X)$ (resp., $\left.C(X)\right)$ extend over $\tilde{X}$ (by the extension theorem for uniform spaces), so $X$ is $C^{*}$ - (resp., $C$-) embedded in $\tilde{X}$; (iii) $\tilde{X}$ is compact (resp., realcompact), by (i).

But it seems worthwhile to indicate, as we have, the details of the construction in Top without reference to Unif. 
Axiom OF CHOICE. We obviously have made free use of $A C$. It is known that $A C$ is needed to construct $\beta X$, and not needed to construct the completion of a uniform space or $v X$. Gillman-Jerison [4] discuss this, and Bourbaki [1] constructs completion without $A C$. Granted completion without $A C$, the method of the previous paragraph yields $v X$ without $A C$, but for $\beta X, A C$ enters via (i). Of course, if one defines $X$ to be compact if $X$ is complete with respect to $C^{*}(X)$, then $A C$ is avoided, but the completion $\tilde{X}$ cannot be shown compact in the usual sense. Comfort [2] has carried out completely an $A C$-free program of this sort.

DiReCT Limits. If $\mathscr{C}$ is one of the chains in the above proof, one makes $\mathscr{C}$ into a direct system by: if $(Y, e) \leqq\left(Y, e^{\prime}\right)$, then take as the bonding map the unique $Y \stackrel{h}{\rightarrow} Y^{\prime}$ with $h \circ e=e^{\prime}$. It is easily seen that $Q$ is the direct limit. In general, the limit of a direct system exists in Tych or Unif (since these categories have sums and quotients) but not much can be said about it without strong properties of the bonding maps. See [3], [5], [6]. In particular, a direct limit in Tych can differ from the direct limit in Top (all topological spaces), the condition for equality being that the latter be in Tych, and in particular, these limits can differ when $(*)$ the system is totally ordered, with all bonding maps dense embeddings [5]. But the above proof shows that if $(*)$ holds, and the bonding maps are $C^{*}$-embeddings, then the direct limit in Top is in Tych, and any member of the system embeds densely. (The proof also shows that in Unif, if a system satisfies (*), then any member of the system embeds densely in the limit.)

\section{REFERENCES}

1. N. Bourbaki, Éléments de mathématique, Fasc. II. Livre III. Topologie générale. Chap. 1: Structures topologiques, Actualités Sci. Indust., no. 1142, Hermann, Paris, 1961; English transl., Hermann, Paris, Addison-Wesley, Reading, Mass., 1966. MR 34 \#5044a; MR 25 \#4480.

2. W. W. Comfort, A theorem of Stone-Cech type, and a theorem of Tychonoff type, without the axiom of choice; and their realcompact analogues, Fund. Math 63 (1968), 97-110. MR 38 \#5174.

3. J. Dugundji, Topology, Allyn and Bacon, Boston, Mass., 1966. MR 33 \#1824.

4. L. Gillman and M. Jerison, Rings of continuous functions, Univ. Series in Higher Math., Van Nostrand, Princeton, N.J., 1960. MR 22 \#6994.

5. D. W. Hajek and G. Strecker, Direct limits of Hausdorff spaces (to appear).

6. H. Herrlich, Separation axioms and direct limits, Canad. Math. Bull. 12 (1969), 337-338. MR 40 \#6487.

7. J. R. Isbell, Uniform spaces, Math. Surveys, no. 12, Amer. Math. Soc., Providence, R.I., 1964. MR 30 \#561.

Department of Mathematics, Wesleyan University, Middletown, Connecticut 06457 